\title{
SOLVENT-DEPENDENT QUENCHING OF THE LOWEST EXCITED SINGLET STATE OF 9,10-DIBROMOANTHRACENE BY GROUND-STATE 2,5-DIMETHYLHEXA-2,4-DIENE YIELDING 9-BROMOANTHRACENE OR THE [4+2] ADDUCT*
}

\author{
T. Nagahara, T. NaKayama and K. HamanouE ${ }^{\dagger}$ \\ Department of Chemistry, Kyoto Institute of Technology \\ Matsugasaki, Sakyo-ku, Kyoto 606-8585, Japan
}

(Received July 23, 1998)

\begin{abstract}
Irrespective of the dielectric constant $(\varepsilon)$ or the hydrogen-bonding ability of a pure solvent, the lowest excited singlet state (DBA*) of 9,10-dibromoanthracene (DBA) is quenched by ground-state 2,5-dimethylhexa-2,4-diene (DMHD) giving rise to the appearance of an exciplex emission. By means of sub-picosecond and nanosecond laser photolysis as well as steady-state photolysis, however, the following solvent-dependent results are obtained; (1) in acetonitrile $(\varepsilon=37.5)$ and acetone $(\varepsilon=20.7)$, an exciplex (DBA-DMHD)* formed between $\mathrm{DBA}^{*}$ and $\mathrm{DMHD}$ generates the DBA radical anion (DBA ${ }^{\circ-}$ ) as an intermediate for formation of 9-bromoanthracene (BA) from DBA; (2) in ethanol $(\varepsilon=24.6)$, 2-propanol $(\varepsilon=19.9)$, 1-octanol $(\varepsilon=10.3)$, diethyl ether $(\varepsilon=4.34)$, and heptane $(\varepsilon=1.92)$, a neutral radical species $\left(\mathrm{NR}^{*}\right)$ generated by decomposition of (DBA-DMHD)* (or by a reaction of $\mathrm{DBA}^{*}$ with DMHD) is an intermediate for formation of a dibenzobicyclo[2.2.2]octadiene-type compound (a [4+2] adduct); (3) both $\mathrm{DBA}^{\bullet-}$ and $\mathrm{NR}^{\bullet}$ are generated in methanol $(\varepsilon=32.7)$ but the rate $(v)$ of BA formation upon steady-state photolysis of DBA in the presence of $1 \mathrm{M}$ DMHD decreases in the order of $v$ (acetonitrile, $\varepsilon=37.5)>v($ acetone, $\varepsilon=20.7)>v$ (methanol, $\varepsilon=32.7$ ). It thus can be concluded that the mechanism of exciplex decomposition and the reaction of $\mathrm{DBA}^{*}$ with $\mathrm{DMHD}$ are affected by not only the dielectric constant of a pure solvent but also its hydrogen-bonding ability.

PACS numbers: 82.40 .Js, $82.50 . \mathrm{Fv}$
\end{abstract}

*The results of this paper were initially presented at The Jablonski Centennial Conference on Luminescence and Photophysics, July 23-27, 1998, Torun, Poland.

tCorresponding author. 


\section{Introduction}

Photoinduced molecular association forming transient ionic species is one of the most important primary processes in a number of photochemical reactions. For example, the amine-assisted dehalogenation of various aromatic halocompounds is well known and the radical anions of halocompounds is generally proposed to be the intermediates [1-9]. By nanosecond laser photolysis and steady-state photolysis of meso-substituted haloanthracenes (the 9-chloro-, 9,10-dichloro-, 9-bromo-, and 9,10-dibromo compounds) in acetonitrile containing $N, N$-dimethylaniline (DMA) or triethylamine (TEA), we have also found that the intermediates for amine-assisted dehalogenation of haloanthracenes yielding 9-haloanthracenes (or anthracene) are really the haloanthracene radical anions [10-12]. By picosecond laser photolysis of 9,10-dibromoanthracene (DBA) in acetonitrile-DMA, furthermore, formation of an exciplex between the lowest excited singlet state ( $\left.\mathrm{DBA}^{*}\right)$ of DBA and ground-state DMA [followed by decomposition into the DBA radical anion $\left(\mathrm{DBA}^{\circ-}\right)$ and the DMA radical cation] is observed [13]. Interestingly, no exciplex formation can be seen in acetonitrile-TEA but nanosecond laser photolysis reveals the existence of $\mathrm{DBA}^{\bullet-}$ as stated previously. Hence, we have proposed that quenching of $\mathrm{DBA}^{*}$ by ground-state TEA in acetonitrile gives rise to formation of a non-emissive short-lived encounter complex or ion pair followed by rapid generation of $\mathrm{DBA}^{\bullet-}$.

The present paper deals with the excited-state dynamics of DBA in several solvents containing 2,5-dimethylhexa-2,4-diene (DMHD) studied by sub-picosecond and nanosecond laser photolysis as well as steady-state photolysis and fluorescence measurements, because the final photoproduct is found to be 9-bromoanthracene (BA) or a dibenzobicyclo[2.2.2] octadiene-type compound (a [4+2] adduct) depending on the nature of a pure solvent.

\section{Experimental}

DBA (Aldrich) was recrystallized three times from ethanol and DMHD (Aldrich) was distilled immediately before use. The solvents used were spectral-grade acetonitrile (Dojin), acetone (Dojin), methanol (Dojin), ethanol (Nacarai), 2-propanol (Dojin), and heptane (Dojin), diethyl ether (Merck) and distilled GR-grade 1-octanol (Wako); although heptane and diethyl ether were used without further purification, all the other solvents were dried using molecular sieves $4 \mathrm{~A}$ (Wako) preheated in a crucible over Bunsen burner and cooled in vacuo. All experiments were performed at room temperature and the sample solutions used for steady-state photolysis and nanosecond laser photolysis in a cell of $10 \mathrm{~mm}$ path length were degassed by several freeze-pump-thaw cycles, while those used for sub-picosecond laser photolysis in a flow cell of $2 \mathrm{~mm}$ path length were deoxygenated by bubbling of Ar gas.

The fluorescence spectra were recorded using a Hitachi MPF-4 spectrofluorometer and steady-state photolysis was carried out using the $404.6 \mathrm{~nm}$ monochromatic light selected from a USH-500D super-high-pressure mercury lamp. The absorption spectral change during photolysis was recorded using a Hitachi 200-20 
spectrophotometer, and the photoproduct formed in heptane-DMHD $(1 \mathrm{M})$ was separated by thin-layer chromatography using a silica gel plate (Merck) and a mixed solvent (hexane/dichloromethane $=4: 1$ in volume ratio) as a developer; the photoproduct thus separated was analyzed using a GE QE-300 ${ }^{1} \mathrm{H}-\mathrm{NMR}$ spectrometer and Wako NMR-grade $\left[{ }^{2} \mathrm{H}\right]$-chloroform as the solvent.

For nanosecond sample excitation, the $355 \mathrm{~nm}$ third harmonic [with a full width at the half-maximum intensity (fwhm) of $5 \mathrm{~ns}$ ] from a Nd ${ }^{3+}$ :YAG laser (Continuum Surelight I) was used and the transient absorption spectra were recorded using a multichannel analyzer [14]; the decay curves of transient absorptions were analyzed by means of a combination of a photomultiplier (Hamamatsu R666) with a storage oscilloscope (Iwatsu TS-8123), and the transient photocurrents were measured by the method reported previously [15].

Sub-picosecond laser photolysis was performed by a pump-probe method using a laser photolysis system comprising a femtosecond mode-locked Ti:sapphire oscillator (Tsunami, model 3960) and a regenerative amplifier (model TSA-50) from Spectra-Physics [16]; both intensities of the excitation light pulse (the $400 \mathrm{~nm}$ second harmonic) and the probing light pulse (the white-light continuum generated by focusing the $800 \mathrm{~nm}$ fundamental into water) were approximated by a Gaussian function $(\mathrm{fwhm}=0.25 \mathrm{ps})$.

\section{Results and discussion}

As shown in Fig. 1a, steady-state photolysis of DBA in acetonitrile-DMHD (1 M) causes the decrement of reactant absorptions (peaks $R_{1}, R_{2}$, and $R_{3}$ ) accompanied by the increment of product absorptions (peaks $P_{1}, P_{2}$, and $P_{3}$ ) which are identical with those of BA. Similar results are also obtained in methanol (and acetone) containing $1 \mathrm{M} \mathrm{DMHD}$ but the rate $(v)$ of BA formation decreases in the order of $v$ (acetonitrile, $\varepsilon=37.5)>v($ acetone, $\varepsilon=20.7)>v$ (methanol, $\varepsilon=32.7$ ), where $\varepsilon$ is the dielectric constant of a pure solvent [17]. For haloanthracene (XA, i.e., the 9-chloro-, 9,10-dichloro-, 9-bromo-, or 9,10-dibromo compound) in acetonitrile, ethanol, cyclohexane, and heptane containing amine (DMA or TEA), XA $\rightarrow$ 9-haloanthracene (or anthracene) dehalogenation can be seen in any solvent but the rate of dehalogenation decreases with the decrease in $\varepsilon$ [10-12]. The intermediates for amine-assisted dehalogenation is concluded to be the XA radical anion ( $\mathrm{XA}^{*-}$ ) on the basis of the following facts; (a) in acetonitrile-amine; at room temperature, nanosecond laser photolysis of XA reveals generation of XA $\mathrm{XA}^{\bullet-}$ within a duration of pulse excitation [10-12]; (b) in acetonitrile without amine at room temperature, pulse radiolysis and $\gamma$ radiolysis of XA reveal generation of $\mathrm{XA}^{\bullet-}$ and formation of 9-haloanthracene (or anthracene), respectively, and $\mathrm{XA}^{\bullet-}$ is also generated by $\gamma$ radiolysis of XA in 2-methyltetrahydrofuran without amine at $77 \mathrm{~K}[11,18]$. In contrast, no photochemical debromination can be seen for DBA in ethanol $(\varepsilon=24.6)$, 2-propanol $(\varepsilon=19.9)$, 1-octanol $(\varepsilon=10.3)$, diethyl ether $(\varepsilon=4.34)$, and heptane $(\varepsilon=1.92)$ containing $1 \mathrm{M}$ DMHD. A typical absorption spectral change caused by steady-state photolysis of DBA in heptane-DMHD (1 M) is shown in Fig. 1b, where the photoproduct is identified to be a dibenzobicyclo[2.2.2]octadiene-type compound (a [4+2] adduct shown in Scheme I) by ${ }^{1} \mathrm{H}-\mathrm{NMR}$ analysis. 


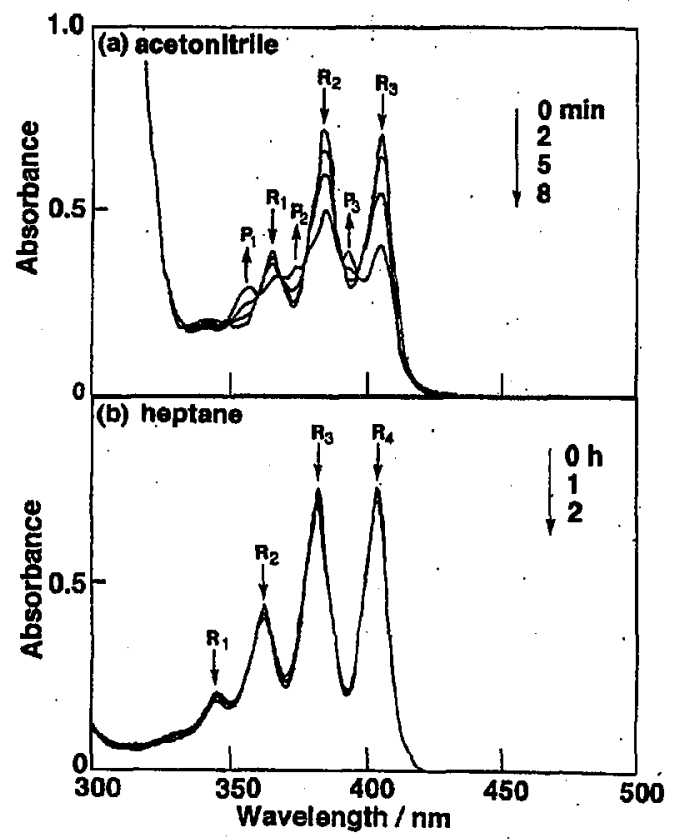

Fig. 1. Absorption spectral changes upon steady-state photolysis of DBA in acetonitrile and heptane containing $1 \mathrm{M}$ DMHD.

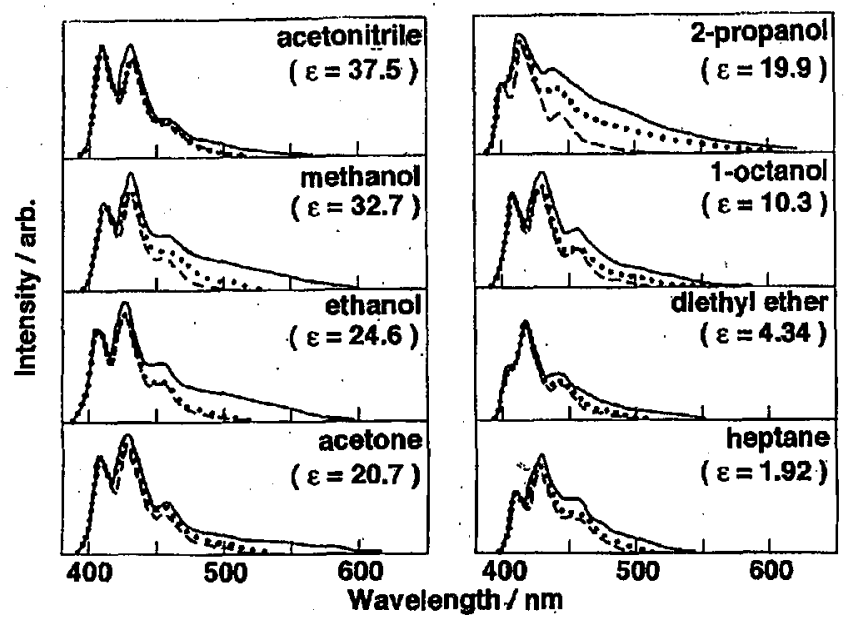

Fig. 2. Monomer (at shorter wavelengths) and exciplex (at longer wavelengths) emission bands recorded for DBA in various solvents containing $0(--), 1(\cdots)$, and 2 (-) M DMHD. The spectral intensities are normalized at the shortest-wavelength emission peak. 
Figure 2 shows the fluorescence spectra recorded for DBA (the dashed line) and DBA-DMHD (the dotted and solid lines). In spite of the solvent-dependent photochemistry of DBA-DMHD stated above, one can clearly observe a broad emission band (undoubtedly, the excimer emission band) at wavelengths longer than those for the structured emission band (the monomer emission band originating from $\left.\mathrm{DBA}^{*}\right)$. The intensities of monomer emissions in the absence $\left(I_{0}\right)$ and presence $\left(I_{\mathrm{D}}\right)$ of DMHD are also measured in all the solvents studied here, and plots of $I_{0} / I_{\mathrm{D}}$ against the concentration of DMHD are found to give the straight lines with slopes $(s)$ listed in Table where the relative yields $(\Phi)$ of exciplex emissions obtained by integration of the several exciplex emission bands over wavenumbers are also listed; by hand-made drawing, estimation of the pure excimer bands is performed.

TABLE

Slope $(s)$, relative yield $(\Phi)$ of exciplex emission, and intermediate (INT) obtained in various solvents containing DMHD.

\begin{tabular}{l|c|c|l}
\hline \hline \multicolumn{1}{c|}{ Solvent } & $s\left[\mathrm{M}^{-1}\right]$ & $\Phi$ & \multicolumn{1}{|c}{ INT } \\
\hline Acetonitrile $(\varepsilon=37.5)$ & 25.7 & 1.0 & $\mathrm{DBA}^{\bullet-}$ \\
Methanol $(\varepsilon=32.7)$ & 17.7 & 3.5 & $\mathrm{NR}^{\bullet} \gg \mathrm{DBA}^{\bullet-}$ \\
Ethanol $(\varepsilon=24.6)$ & 12.4 & 3.6 & $\mathrm{NR}^{\bullet}$ \\
Acetone $(\varepsilon=20.7)$ & 18.5 & 2.4 & $\mathrm{DBA}^{\bullet-}$ \\
2-Propanol $(\varepsilon=19.9)$ & 10.4 & 3.9 & $\mathrm{NR}^{\bullet}$ \\
1-Octanol $(\varepsilon=10.3)$ & 4.8 & - & $\mathrm{NR}^{\bullet}$ \\
Diethyl ether $(\varepsilon=4.34)$ & 5.4 & 3.9 & $\mathrm{NR}^{\bullet}$ \\
Heptane $(\varepsilon=1.92)$ & 5.3 & 10.1 & $\mathrm{NR}^{\bullet}$
\end{tabular}

By nanosecond laser photolysis of DBA in acetonitrile, acetone, and methanol containing $1 \mathrm{M}$ DMHD, the transient absorption spectra (the solid lines) shown in Fig. 3 can be seen even at the end of pulse excitation, i.e., $10 \mathrm{~ns}$ delay. In comparison with the normalized absorption spectrum (the dotted line) of the DBA radical anion ( $\mathrm{DBA}^{\bullet-}$ ) obtained by nanosecond laser photolysis of DBA in acetonitrile-TEA [12], it is clear that $\mathrm{DBA}^{\bullet-}$ is generated in acetonitrile-DMHD (and acetone-DMHD) but a transient species with an absorption band somewhat different from that of $\mathrm{DBA}^{\bullet-}$ is generated in methanol-DMHD. This is supported by the results shown in Fig. 4, i.e., the lifetimes $(\tau)$ of transient absorptions (a) and photocurrents (b) are identical in acetonitrile and acetone containing DMHD, but the transient photocurrent in methanol-DMHD is too weak to record its decay curve.

As stated previously, the absorption spectral changes upon steady-state photolysis of DBA in ethanol $(\varepsilon=24.6)$, 2-propanol $(\varepsilon=19.9)$, 1-octanol $(\varepsilon=10.3)$, diethyl ether $(\varepsilon=4.34)$, and heptane $(\varepsilon=1.92)$ containing 1 M DMHD are identical, and formation of a dibenzobicyclo[2.2.2]octadiene-type compound (a [4+2] adduct) is confirmed in heptane-DMHD. In accordance with this, Fig. 5 indicates 


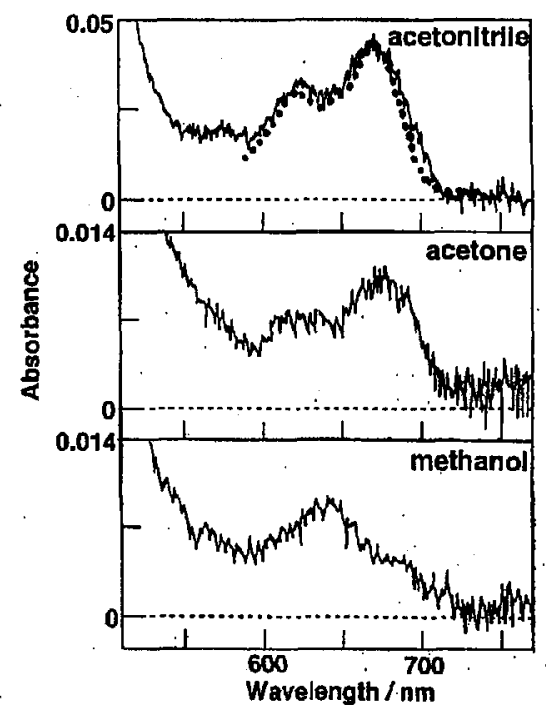

Fig. 3. Transient absorption spectra $(-)$ at 10 ns delay obtained by nanosecond laser photolysis of DBA in acetonitrile, acetone, and methanol containing $1 \mathrm{M}$ DMHD. The dotted spectrum is due to the absorption of $\mathrm{DBA}^{--}$obtained by nanosecond laser photolysis of DBA in acetonitrile-TEA [12].

that the transient absorption spectra obtained by nanosecond laser photolysis of DBA in the solvents stated above are clearly different from the absorption spectrum (the dotted line shown in Fig. 3) of DBA -- , and, as shown in Fig. 6 as typical examples, the transient absorptions in ethanol-DMHD and heptane-DMHD still decrease following the first-order reaction kinetics; these results are similar to those obtained in methanol-DMHD (cf. Figs. 3 and 4). Formation of a [4+2] adduct was also observed upon steady-state photolysis of 9,10-dichloroanthracene (DCA) in heptane-DMHD by us [19] and in benzene-DMHD by Saltiel et al. [20] who proposed a neutral radical species (essentially identical with $\mathrm{NR}^{\bullet}$ shown in Scheme I) as an intermediate. Hence, the lifetimes $(\tau)$ of transient absorptions indicated in Fig. 6 and that obtained in methanol-DMHD (cf. Fig. 4) may correspond to the time constants for conversion of $\mathrm{NR}^{\bullet}$ to a [4+2] adduct. Taking into account of the solvent-dependent generation of an intermediate (DBA ${ }^{\bullet}$ or $\mathrm{NR}^{\bullet}$ ) stated so far, a qualitative interpretation of the results listed in Table may be that the relatively large and small values of $s$ and $\Phi$, respectively, reflect enhanced generation of $\mathrm{DBA}^{\bullet-}$ (and thus suppressed generation of $\mathrm{NR}^{\bullet}$ ).

Figure 7 shows the transient absorption spectra obtained by sub-picosecond laser photolysis of DBA in acetonitrile-DMHD(1 M); a similar result is obtained in acetone-DMHD $(1 \mathrm{M}$ ). Clearly, band $A$ (due to the singlet-singlet absorption of $\mathrm{DBA}^{*}$ [13]) appears at first and its decrement with time is accompanied by the increment and then the decrement of band $B$; after its disappearance, band $C$ can be seen as a residual absorption band which can be ascribed to the absorption of $\mathrm{DBA}^{--}$in comparison with the transient absorption spectrum obtained by 


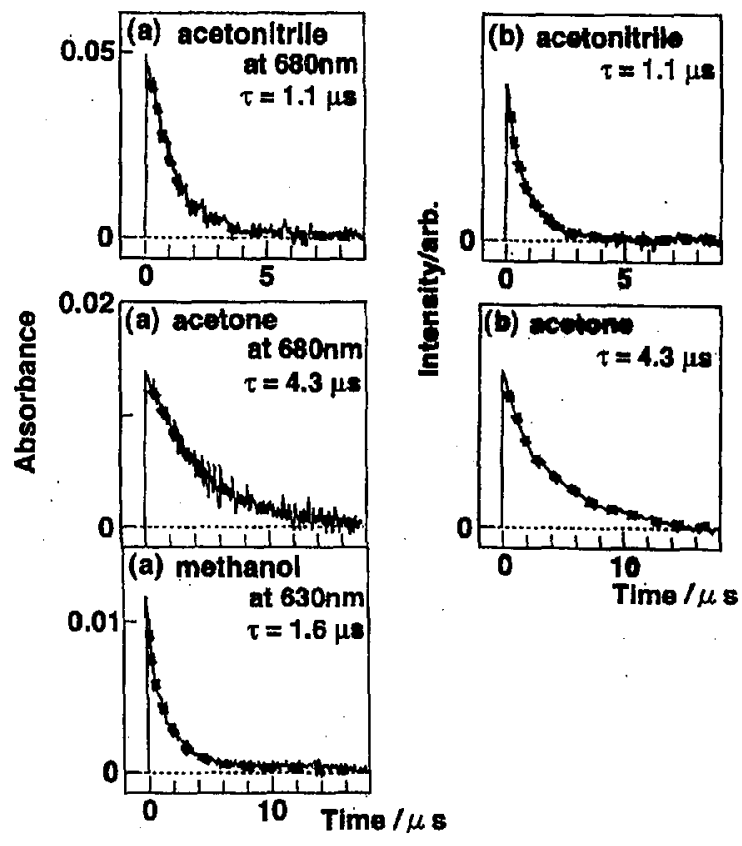

Fig. 4. Decay curves (-) of transient absorptions (a) and photocurrents (b) obtained by nanosecond laser photolysis of DBA in acetonitrile, acetone, and methanol containing 1 M DMHD. The dashed curves are the best-fit single-exponential decay ones with lifetimes $(\tau)$ indicated.

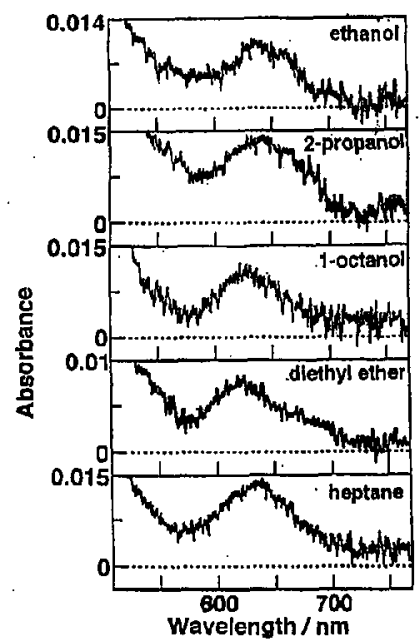

Fig. 5. Transient absorption spectra at $10 \mathrm{~ns}$ delay obtained by nanosecond laser photolysis of DBA in ethanol, 2-propanol, 1-octanol, diethyl ether, and heptane containing 1 M DMHD. 


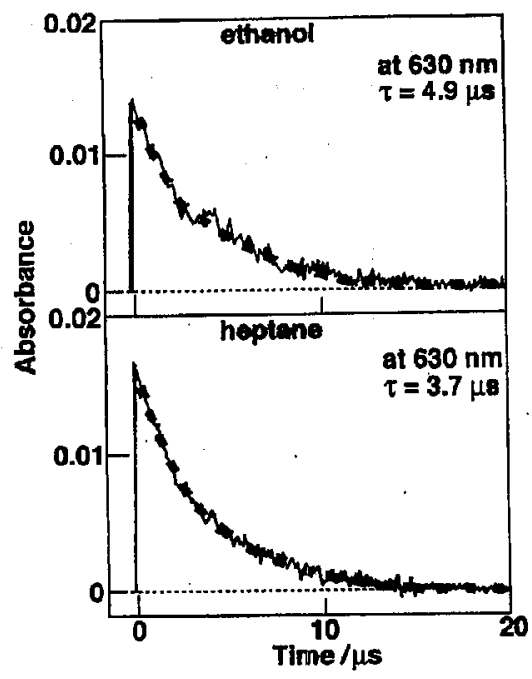

Fig. 6. Decay curves (-) of transient absorptions obtained by nanosecond laser photolysis of DBA in ethanol and heptane containing $1 \mathrm{M}$ DMHD. The dashed curves are the best-fit single-exponential decay ones with lifetimes $(\tau)$ indicated.

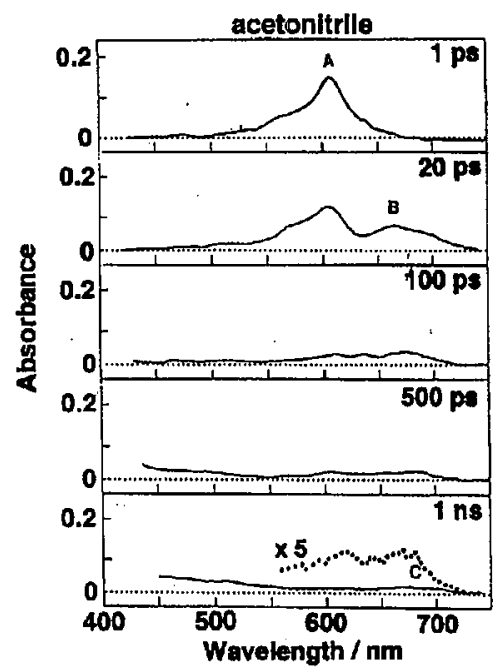

Fig. 7. Transient absorption spectra obtained by sub-picosecond laser photolysis of DBA in acetonitrile-DMHD $(1 \mathrm{M})$.

nanosecond laser photolysis of DBA in acetonitrile-DMHD (cf. Fig. 3). As shown in Fig. 8, furthermore, the absorbance changes of bands $A\left[D_{A}(t) / D_{A}(\max )\right.$, open circles $]$ and $B\left[D_{B}(t) / D_{B}(\max )\right.$, closed circles] with time can well be reproduced by the solid curves. These curves are calculated as follows; (i) a Gaussian intensity function with fwhm $=0.25 \mathrm{ps}$ is assumed for both the excitation and probing light 


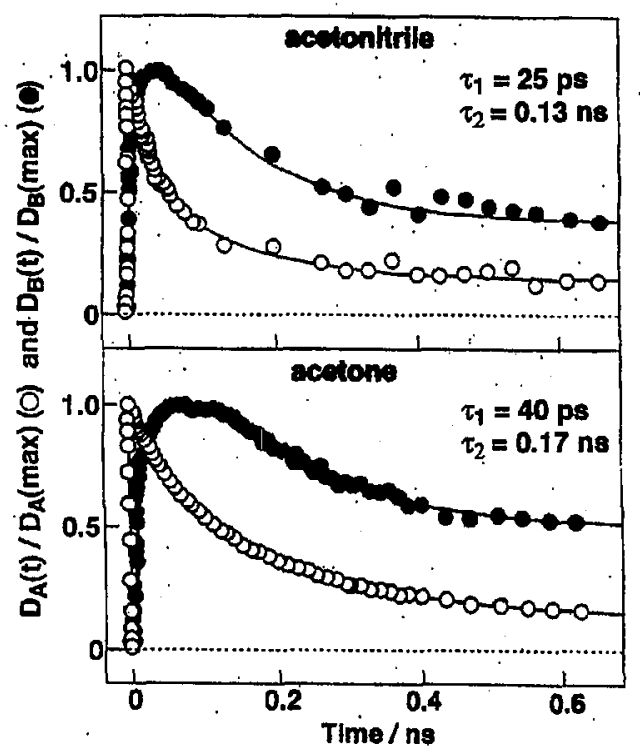

Fig. 8. $D_{A}(t) / D_{A}(\max )$ [monitored at $\left.610 \mathrm{~nm}(0)\right]$ and $D_{B}(t) / D_{B}(\max )$ [monitored at $680 \mathrm{~nm}(\bullet)$ ] obtained in acetonitrile and acetone containing $1 \mathrm{M}$ DMHD. The solid curves (with time constants $\tau_{1}$ and $\tau_{2}$ ) are the best-fit absorbances calculated for the superposition of absorptions due to $\mathrm{DBA}^{*},(\mathrm{DBA}-\mathrm{DMHD})^{*}$, and $\mathrm{DBA}^{\bullet-}$ with concentration functions given by Eqs. (1)-(3), respectively.

pulses; (ii) the concentrations of $\mathrm{DBA}^{*}\left[C_{A}(t)\right]$, a transient species $\left[C_{E}(t)\right]$, and $\mathrm{DBA}^{\bullet-}\left[C_{I}(t)\right]$ are expressed by biexponential functions (with time constants $\tau_{1}$ and $\tau_{2}$ ) given by Eqs. (1)-(3), respectively,

$$
\begin{aligned}
& C_{A}(t)=c_{1} \exp \left(-t / \tau_{1}\right)+c_{2} \exp \left(-t / \tau_{2}\right), \\
& C_{E}(t)=c_{3}\left[-\exp \left(-t / \tau_{1}\right)+\exp \left(-t / \tau_{2}\right)\right], \\
& C_{I}(t)=-c_{4}\left[1-\exp \left(t / \tau_{1}\right)\right]+c_{5}\left[1-\exp \left(-t / \tau_{2}\right)\right],
\end{aligned}
$$

where $c_{1}-c_{5}$ are the positive experimental constants; (iii) both bands $A$ and $B$ are the superposition of absorptions due to $\mathrm{DBA}^{*}$, a transient species, and DBA ${ }^{\bullet-}$. Hence, band $B$ can undoubtedly be ascribed to the absorption of an exciplex [(DBA-DMHD $\left.)^{*}\right]$ formed between DBA* and DMHD. In fact, the absorption band $(B)$ shown in Fig. 7 and the broad emission band shown in Fig. 2 are similar to those observed for an exciplex formed between $\mathrm{DBA}^{*}$ and amine (DMA or TEA) in acetonitrile, ethanol, and heptane [13]. As shown in Fig. 9, however, only band $A$ can be seen by sub-picosecond laser photolysis of DBA in ethanol-DMHD $(1 \mathrm{M})$; a similar result is obtained in heptane-DMHD $(1 \mathrm{M})$. In spite of this circumstance, Fig. 10 indicates that band $A$ observed in ethanol or heptane containing $1 \mathrm{M}$ DMHD still decreases with time following a biexponential function. Probably, the absorption band of $\mathrm{DBA}^{*}$ may be superposed by that of (DBA-DMHD)* , because the excimer emission is observed in all the solvents studied here and band $A$ shown in Fig. 9 is slightly broad compared with that obtained in the absence of DMHD. 


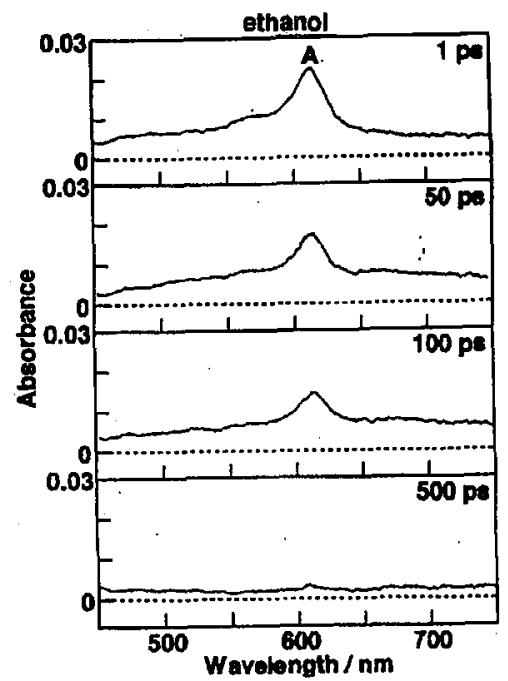

Fig. 9. Transient absorption spectra obtained by sub-picosecond laser photolysis of DBA in ethanol-DMHD(1 M).

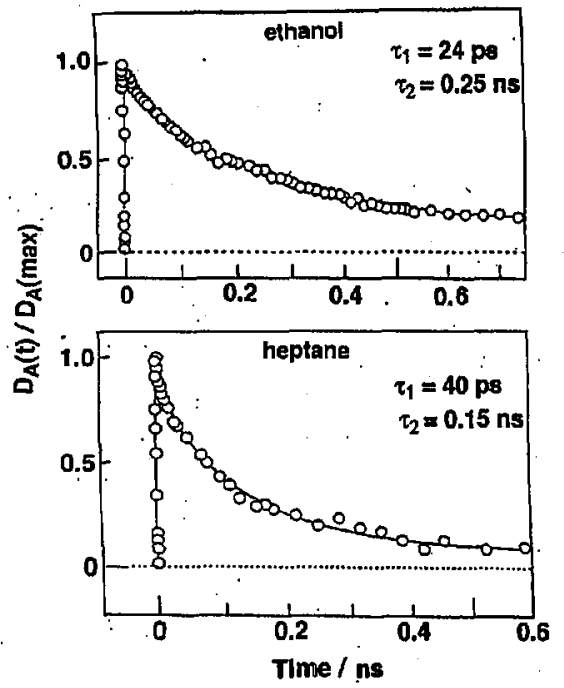

Fig. 10. $D_{A}(t) / D_{A}(\max )$ [monitored at $610 \mathrm{~nm}(0)$ ] obtained in ethanol and heptane containing $1 \mathrm{M}$ DMHD. The solid curves (with time constants $\tau_{1}$ and $\tau_{2}$ ) are the best-fit absorbances calculated for the superposition of absorptions due to $\mathrm{DBA}^{*}$ and (DBA-DMHD)* with concentration functions given by Eqs. (1) and (2), respectively.

Saltiel et al. [20] proposed that an exciplex formed between the lowest excited singlet state $\left(\mathrm{DCA}^{*}\right)$ of $\mathrm{DCA}$ and ground-state DMHD in benzene generated a neutral radical species essentially identical with $\mathrm{NR}^{\bullet}$ ) which was the precursor for 
formation of a [4+2] adduct. If this is true, the results obtained so far may be summarized as follows (cf. the processes shown by solid arrows in scheme I [21]):

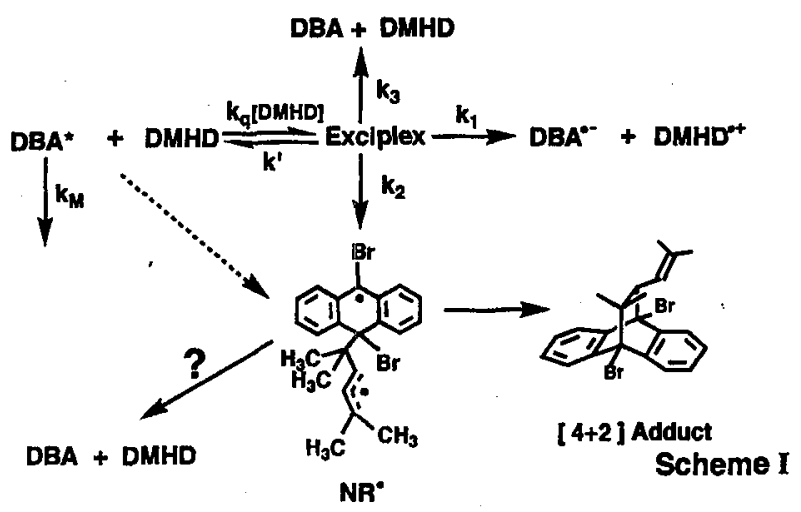

(1) (DBA-DMHD)* formed in acetonitrile $(\varepsilon=37.5)$ or acetone $(\varepsilon=20.7)$ decomposes into DBA - and the DMHD radical cation $\left(\mathrm{DMHD}^{\bullet+}\right)$, and $\mathrm{DBA}^{\bullet-}$ thus generated is an intermediate for DMHD-assisted DBA $\rightarrow$ BA debromination; although Saltiel et al. $[22,23]$ reported formation of a DCA-DMHD exciplex (by direct excitation of a ground-state DCA-DMHD complex) or a triplex (by a reaction of a DCA-DMHD exciplex with DMHD), no such evidences were obtained by our previous study [19]. (2) (DBA-DMHD)* formed in ethanol $(\varepsilon=24.6)$, 2-propanol $(\varepsilon=19.9)$, 1-octanol $(\varepsilon=10.3)$, diethyl ether $(\varepsilon=4.34)$, and heptane $(\varepsilon=1.92)$ generates a neutral radical species $\left(\mathrm{NR}^{\bullet}\right)$ as an intermediate for formation of a [4+2] adduct; since [4+2] addition of DMHD to $\mathrm{DBA}^{*}$ is forbidden [24], the existence of $N^{*}$ is reasonable but its decomposition into the original compounds (DBA and DMHD) may be a major process owing to the extremely slow photochemical reaction of DBA (cf. Fig. 1b). (3) Both DBA ${ }^{\bullet-}$ (minor) and $\mathrm{NR}^{\bullet}$ (major) are generated in methanol $(\varepsilon=32.7)$, because steady-state photolysis reveals $\mathrm{DBA} \rightarrow \mathrm{BA}$ debromination but the transient absorption spectrum obtained by nanosecond laser photolysis can be ascribed to the absorption of $\mathrm{NR}^{\bullet}$ superposed by the weak absorption of DBA ${ }^{\bullet-}$.

Denoting the sum of rate constants $\left(\mathrm{k}_{1}, \mathrm{k}_{2}\right.$, and $\mathrm{k}_{3}$ shown in Scheme I) by $k_{E}$, the sum of the inverses of time constants ( $\tau_{1}$ and $\tau_{2}$ obtained previously) should be expressed by

$$
1 / \tau_{1}+1 / \tau_{2}=k_{q}[\mathrm{DMHD}]+k^{\prime}+k_{E}+k_{M},
$$

where [DMHD] is the concentration of DMHD and $k_{M}$ is the decay rate constant of, DBA $^{*}$ obtained in the absence of DMHD. The rate constants $k_{q}$ and $k_{E}^{\prime}=k^{\prime}+k_{E}$ can thus be obtained from the slope and the intercept of Eq. (4), respectively. By measurements of the time-dependent intensity changes of bands $A$ and $B$ at various DMHD concentrations in acetonitrile and ethanol, the time constants $\left(\tau_{1}\right.$ and $\tau_{2}$ ) are determined and a plot of $1 / \tau_{1}+1 / \tau_{2}$ against DMHD concentration gives a straight line (cf. Fig. 11). Denoting the efficiency $\left[k^{\prime} /\left(k^{\prime}+k_{E}\right)\right]$ for re-population of $\mathrm{DBA}^{*}$ from (DBA-DMHD)* by $\gamma$, the values of $s$ listed in Table can easily be 


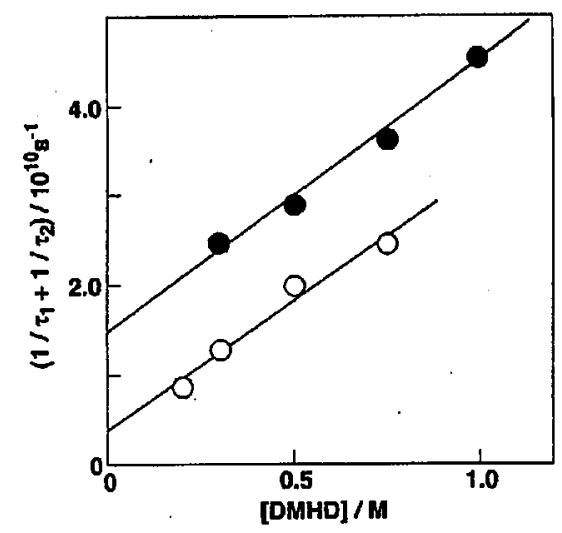

Fig. 11. Plots of $1 / \tau_{1}+1 / \tau_{2}$ against the DMHD concentration in acetonitrile $(0)$ and ethanol (•).

found to be equal to $k_{q}(1-\gamma) / k_{M}$ and thus a combination of $\gamma$ (derived from $s, k_{q}=2.8 \times 10^{10} \mathrm{M}^{-1} \mathrm{~s}^{-1}$ in acetonitrile or $3.0 \times 10^{10} \mathrm{M}^{-1} \mathrm{~s}^{-1}$ in ethanol, and $k_{M}=7.7 \times 10^{8} \mathrm{~s}^{-1}$ in acetonitrile or $7.1 \times 10^{8} \mathrm{~s}^{-1}$ in ethanol [13]) with $k_{E}^{\prime}=k^{\prime}+k_{E}$ gives the decay rate constants $\left(k^{\prime}\right.$ and $k_{E}$ ) of (DBA-DMHD)* . In acetonitrile, $k^{\prime}=1.4 \times 10^{10} \mathrm{~s}^{-1}$ and $k_{E}=3.1 \times 10^{9} \mathrm{~s}^{-1}$ obtained are comparable with those $\left(k^{\prime}=\right.$ $1.5 \times 10^{9} \mathrm{~s}^{-1}$ and $k_{E}=1.1 \times 10^{9} \mathrm{~s}^{-1}$ ) estimated for an exciplex (formed between $\mathrm{DCA}^{*}$ and DMHD) on an assumption of diffusion-controlled quenching $\left(k_{q}=\right.$ $\left.1.9 \times 10^{10} \mathrm{M}^{-1} \mathrm{~s}^{-1}\right)$ of $\mathrm{DCA}^{*}$ by DMHD [19] as well as those $\left(k^{\prime}=2.7 \times 10^{9} \mathrm{~s}^{-1}\right.$ and $k_{E}=4.8 \times 10^{9} \mathrm{~s}^{-1}$ ) of an exciplex formed between DBA* and DMA [13]. $k_{q}$ (stated previously) and $k_{E}=3.8 \times 10^{9} \mathrm{~s}^{-1}$ obtained for (DBA-DMHD) ${ }^{*}$ in ethanol are also comparable with those obtained in acetonitrile, but $k^{\prime}=9.4 \times 10^{9} \mathrm{~s}^{-1}$ obtained in the former solvent is about 6.7-times greater than that obtained in the latter solvent. Hence, the mechanism of exciplex decomposition may be affected by not only the dielectric constant of a pure solvent but also its hydrogen-bonding ability.

Although we have proposed competing generation of $\mathrm{DBA}^{\bullet-}$ and $\mathrm{NR}^{\bullet}$ from $(\mathrm{DBA}-\mathrm{DMHD})^{*}$ following the mechanism for a [4+2] adduct formation reported by Saltiel et al. [20], no definitive information reflecting generation of $\mathrm{NR}^{*}$ from $(\mathrm{DBA}-\mathrm{DMHD})^{*}$ is presently obtained. Hence, an alternative mechanism is that $\mathrm{NR}^{\bullet}$ is generated by a diffusion-controlled reaction of $\mathrm{DBA}^{*}$ with DMHD (cf. the process shown by the dashed arrow in Scheme I). Experimental works along this line are in progress.

\section{Acknowledgment}

This work was supported by a Grant-in-Aid for Priority-Area-Research on Photoreaction Dynamics from the Ministry of Education, Science, Sports and Culture of Japan (No. 06239101 and 06239106). 


\section{References}

[1] M. Ohashi, K. Tsujimoto, K. Seki, J. Chem. Soc. Chem. Commun., 384 (1973).

[2] K. Tsujimoto, S. Tasaka, M. Ohashi, J. Chem. Soc. Chem. Commun, 758 (1975).

[3] N.J. Bunce, S. Safe, L.O. Ruzo, J. Chem. Soc. Perkin Trans. 1, 1607 (1975).

[4] N.J. Bunce, P. Pilon, L.O. Ruzo, D.J. Sturch, J. Org. Chem. 41, 3023 (1976).

[5] N.J. Bunce, Y. Kumar, L. Ravanal, S. Safe, J. Chem. Soc. Perkin Trans. 2, 880 (1978).

[6] B. Chittim, S. Safe, N. Bunce, L.O. Ruzo, K. Olie, O. Hutzinger, Can. J. Chem. 56, 1253 (1978).

[7] R.S. Davidson, J.W. Goodwin, Tetrahedron Lett. 22, 163 (1981).

[8] F.D. Saeva, Topics Current Chem. 156, 61 (1990).

[9] J. Fulara, T. Latowski, Polish J. Chem. 64, 369 (1990).

[10] K. Hamanoue, S. Tai, T. Hidaka, T. Nakayama, M. Kimoto, H. Teranishi, J. Phys. Chem. 88, 4380 (1984).

[11] K. Hamanoue, T. Nakayama, K. Ikenaga, K. Ibuki, J. Phys. Chem. 96, 10297 (1992).

[12] K. Hamanoue, T. Nakayama, K. Ikenaga, K. Ibuki, A. Otani, J. Photochem. Photobiol. A: Chem. 69, 305 (1993).

[13] T. Nakayama, T. Hamana, P. Jana, S. Akimoto, I. Yamazaki, K. Hamanoue, J. Phys. Chem. 100, 18431 (1996).

[14] K. Ushida, T. Nakayama, T. Nakazawa, K. Hamanoue, T. Nagamura, A. Mugishima, S. Sakimukai, Rev. Sci. Instrum. 60, 617 (1989).

[15] K. Hamanoue, T. Nakayama, S. Asada, K. Ibuki, J. Phys. Chem. 96, 3736 (1992).

[16] T. Nakayama, Y. Amijima, K. Ibuki, K. Hamanoue, Rev. Sci. Instrum. 68, 4364 (1997).

[17] J.A. Riddick, W.B. Bunger, Technique of Chemistry, Vol. II, Organic Solvents, 3rd ed., Wiley-Interscience, New York 1970.

[18] K. Hamanoue, M. Kimoto, T. Nakayama, H. Teranishi, S. Tagawa, Y: Tabata, Radiat. Phys. Chem. 24, 445 (1984).

[19] T. Nakayama, Y. Amijima, S. Miki, K. Hamanoue, Chem. Lett., 223 (1997).

[20] J. Saltiel, R. Dabestani, D.F. Sears, Jr., W.M. McGowan, E.F. Hilinski, J. Am. Chem. Soc. 117, 9129 (1995).

[21] J.B. Birks, Photophysics of Aromatic Molecules, Wiley-Interscience, New York 1970, Ch. 7.

[22] J. Saltiel, D.E. Townsend, B.D. Watson, P. Shannon, J. Am. Chem. Soc. 97, 5688 (1975).

[23] W.K. Smothers, K.S. Schange, J. Saltiel, J. Am. Chem. Soc. 101, 1895 (1979).

[24] R.B. Woodward, R. Hoffman, Angew. Chem. Int. Ed. Engl. 8, 781 (1969). 